\title{
On isomorphisms between deformation rings and Hecke rings
}

Dedicated to the memory of my mother Nalini B. Khare

30th August 1936-12th March 2002

Chandrashekhar Khare

\section{Introduction}

It was proven in [W] and [TW] that in many cases there is an isomorphism between certain deformation rings and Hecke rings associated to an irreducible, modular, $\bmod p$ representation

$$
\bar{\rho}: G_{\mathbf{Q}} \rightarrow G L_{2}(k)
$$

with $k$ a finite field of characteristic $p \geq 3$ and $G_{\mathbf{Q}}$ the absolute Galois group of $\mathbf{Q}$. As is well-known, the proven cases were enough to establish the modularity of semistable elliptic curves. There were 2 steps in the proof:

1. Establish an isomorphism between the minimal deformation ring and minimal Hecke ring (defined below as $R_{\phi}$ and $\mathbf{T}_{\phi}$ ): this was done in [TW] using what are called Taylor-Wiles (TW) systems now.

2. Deduce an isomorphism between non-minimal deformation rings and Hecke rings using a numerical criterion established in [W] for maps between certain rings to be isomorphisms of complete intersections and results about raising levels of newforms: this was done in [W].

In this article we give a different approach to establishing the isomorphisms between deformation rings and Hecke rings proved in [W] and [TW] in the case when $\bar{\rho}$ is semistable that does not use TW systems. While the approach here follows the over-all strategy of Wiles, instead of the first step above we use the isomorphism of certain new quotients $\left(R_{Q}^{Q-\text { new }} \simeq \mathbf{T}_{Q}^{Q-\text { new }}\right.$ : 
see Theorem 1 below) of the deformation rings and Hecke rings (denoted by $R_{Q}$ and $\mathbf{T}_{Q}$ below) that one would like to prove isomorphic: this isomorphism results from the recent Galois cohomological work of R. Ramakrishna in $[R]$ and $[\mathrm{R} 1]$ and the level raising results of F. Diamond and R. Taylor in [DT] and [DT1]. After this the lifting of the isomorphism $R_{Q}^{Q-\text { new }} \simeq \mathbf{T}_{Q}^{Q-\text { new }}$ to an isomorphism $R_{Q} \simeq \mathbf{T}_{Q}$ is carried out by applying the level lowering results of [RT] (see Theorem 4 below), and the numerical criterion alluded to above of Wiles as refined by Lenstra (see Theorem 3 below). In the last section we sketch another approach that uses a "level substitution mod $p^{n}$ " trick that has some novelty.

\section{Deformation rings and Hecke rings}

Let $\bar{\rho}: G_{\mathbf{Q}} \rightarrow G L_{2}(k)$ be continuous and irreducible mod $p$ representation with $k$ a finite field of characteristic $p>5$ which satisfies the following conditions:

- $\bar{\rho}$ is modular, i.e., arises from a newform of some level $N$ and weight $k \geq 2$ with respect to an embedding $\iota_{p}: \overline{\mathbf{Q}} \rightarrow \overline{\mathbf{Q}_{p}}$ that we fix.

- $\operatorname{det}(\bar{\rho})=\omega$ the $\bmod p$ cyclotomic character.

$$
\left.\bar{\rho}\right|_{G_{\ell}} \simeq\left(\begin{array}{cc}
\omega \chi^{-1} & * \\
0 & \chi
\end{array}\right)
$$

for $\chi$ an unramified character of $G_{\ell}$, for $\ell(\neq p) \in S$ with $S$ the set of places at which $\bar{\rho}$ is ramified (we then have $\chi^{2}=1$ ) and with $G_{\ell}$ a decomposition group at $\ell$.

- $\left.\bar{\rho}\right|_{G_{p}}$ is either finite, or not finite but Selmer, i.e., of the form

$$
\left(\begin{array}{cc}
\omega \psi^{-1} & * \\
0 & \psi
\end{array}\right)
$$

for an unramified character $\psi$, with the further condition that its restriction to the inertia subgroup is not the generic fibre of a finite flat group scheme over the ring of integers of the maximal unramified extension of $\mathbf{Q}_{p}$ (we then have $\psi^{2}=1$ ). 
Note that this implies that $\operatorname{Ad}^{0}(\bar{\rho})$ is absolutely irreducible and the order of $\operatorname{im}(\bar{\rho})$ is divisible by $p$ (otherwise $\bar{\rho}$ has Serre weight 2 and Artin conductor 1 by assumptions on determinant and ramification above) which give the properties of $\bar{\rho}$ required in $[R]$ and $[R 1]$ (we thank the referee for pointing this out).

\subsection{Deformation rings}

We briefly recall the existence of certain deformation rings parametrising lifts of $\bar{\rho}$ with given local conditions, referring to $[\mathrm{Ma}]$ and $[\mathrm{KR}]$ for more details. We denote by $\varepsilon$ the $p$-adic cyclotomic character. For a complete Noetherian local $W(k)$-algebra $R$ with residue field $k$, a representation $\rho: G_{\mathbf{Q}} \rightarrow G L_{2}(R)$ that reduces modulo the maximal ideal to give $\bar{\rho}$, is said to be minimally ramified at at a prime $\ell$ if:

1. $\ell$ is not in $S$, then $\rho$ is unramified at $\ell$

2. $\ell \in S$ and $\ell \neq p$, then if we denote by $I_{\ell}$ an inertia subgroup at $\ell,\left.\rho\right|_{I_{\ell}}$ is isomorphic to

$$
\left(\begin{array}{ll}
1 & * \\
0 & 1
\end{array}\right)
$$

3. if $\ell=p$ then $\left.\rho\right|_{I_{p}}$ is finite if $\bar{\rho}$ is finite, and is otherwise of the form

$$
\left(\begin{array}{ll}
\varepsilon & * \\
0 & 1
\end{array}\right)
$$

For a finite set of primes $Q$, not congruent to $\pm 1 \bmod p$, at which $\bar{\rho}$ is unramified, and $\left.\bar{\rho}\right|_{G_{q}}$ is of the form

$$
\pm\left(\begin{array}{ll}
\varepsilon & 0 \\
0 & 1
\end{array}\right)
$$

consider the ring $R_{Q}^{Q-\text { new }}$ (that is called $R_{S \cup Q}^{Q-\text { new }}$ in $[\mathrm{KR}]$ ) that is the universal ring that parametrises (isomorphism classes of) lifts of $\bar{\rho}$ to $G L_{2}(R)$ for complete Noetherian local $W(k)$-algebras $R$ with residue field $k$, such that these are

- Unramified outside $S \cup Q$ 
- Minimally ramified, in the above sense, at primes in the set $S$ that consists of the ramified primes of $\bar{\rho}$

- At primes $q \in Q$ on restriction to $G_{q}$ have the form

$$
\pm\left(\begin{array}{ll}
\varepsilon & * \\
0 & 1
\end{array}\right)
$$

- With determinant $\varepsilon$, the $p$-adic cyclotomic character.

Such a universal ring $R_{Q}^{Q-\text { new }}$, that is a complete Noetherian local $W(k)$ algebra with residue field $k$, exists as the conditions above are conditions in the sense of Section 23 of [Ma] because of our assumption that the primes in $Q$ are not congruent to $1 \bmod p$ (see Lemma 2 of $[\mathrm{KR}]$ for more details), and we denote the corresponding universal representation $G_{\mathbf{Q}} \rightarrow G L_{2}\left(R_{Q}^{Q-\text { new }}\right)$ by $\rho_{Q}^{Q-\text { new }}$. If we delete the third condition on primes in $Q$ then we denote the corresponding ring $R_{Q}$. We have the natural surjective map $R_{Q} \rightarrow R_{Q}^{Q-\text { new }}$ of local $W(k)$-algebras. More generally for a subset $\alpha$ of $Q$ if we impose the third condition only for primes in $\alpha$ then we denote the corresponding deformation ring by $R_{Q}^{\alpha-n e w}$ and the corresponding universal representation $G_{\mathbf{Q}} \rightarrow G L_{2}\left(R_{Q}^{\alpha-\text { new }}\right)$ by $\rho_{Q}^{\alpha-\text { new }}:$ we have surjective maps $R_{Q} \rightarrow R_{Q}^{\alpha-\text { new }} \rightarrow$ $R_{Q}^{Q-\text { new }}$ of local $W(k)$-algebras. If $Q$ is empty we denote the corresponding ring by $R_{\phi}$ and call it the minimal deformation ring, and denote by $\rho_{\phi}$ the corresponding universal representation.

We denote by $G_{S \cup Q}$ the Galois group of the maximal extension of $\mathbf{Q}$ in $\overline{\mathbf{Q}}$ unramified outside $S$ and $Q$ and the infinite place.

Definition 1 The Selmer group $H_{Q}^{1}\left(G_{S \cup Q}, \operatorname{Ad}^{0}(\bar{\rho})\right)$ is the kernel of the restriction map

$$
H^{1}\left(G_{S \cup Q}, \operatorname{Ad}^{0}(\rho)\right) \rightarrow \oplus_{v \in S \cup Q} H^{1}\left(G_{v}, \operatorname{Ad}^{0}(\bar{\rho})\right) / \mathcal{N}_{v}
$$

Here for $v \neq p$ and $v \in S, \mathcal{N}_{v}$ is described as the image under the inflation map of $H^{1}\left(G_{v} / I_{v}, \operatorname{Ad}^{0}(\bar{\rho}) / \operatorname{Ad}^{0}(\bar{\rho})^{I_{v}}\right)$. For $v=p$ we define it to be either $H_{S e}^{1}\left(G_{p}, \operatorname{Ad}^{0}(\bar{\rho})\right)$ or $H_{f l}^{1}\left(G_{p}, \operatorname{Ad}^{0}(\bar{\rho})\right)$, according to whether $\left.\bar{\rho}\right|_{I_{p}}$ is not finite or it is finite, using the notation of Section 4.1 of [deS]. For $v \in Q$, $\mathcal{N}_{v}$ is described as the subspace of $H^{1}\left(G_{v}, \operatorname{Ad}^{0}(\bar{\rho})\right)$ generated by the cocycle that (in a suitable choice of basis of $V_{\bar{\rho}}$, the 2 -dimensional $k$-vector space that 
affords $\bar{\rho}$, and viewing $\operatorname{Ad}^{0}(\bar{\rho})$ as a subspace of $\left.\operatorname{End}\left(V_{\bar{\rho}}\right)\right)$ sends $\sigma \rightarrow 0$ and $\tau$ to

$$
\left(\begin{array}{ll}
0 & 1 \\
0 & 0
\end{array}\right)
$$

where $\sigma$ and $\tau$ generate the tame quotient of $G_{q}$, and satisfy the relation $\sigma \tau \sigma^{-1}=\tau^{q}$ (this is the "null cocycle" denoted by $s_{q}$ in Section 3 of $[\mathrm{R}]$ ).

Proposition 1 If $\mathrm{m}_{\mathrm{Q}}$ is the maximal ideal of $R_{Q}^{Q-n e w}$, then we have an isomorphism

$$
H_{Q}^{1}\left(G_{S \cup Q}, \operatorname{Ad}^{0}(\bar{\rho})\right) \simeq \operatorname{Hom}\left(\mathrm{m}_{Q} /\left(\mathrm{m}_{Q}^{2}, p\right), k\right) .
$$

Proof. The proof is routine and follows from the standard identification of the cotangent space of deformation rings with the corresponding Selmer groups (see Theorem 15 of [deS] for instance or [Ma1] in [FLT]).

\section{$2.2 \quad$ Hecke rings}

Let $N(\bar{\rho})$ be the prime to $p$ part of the Artin conductor of $\bar{\rho}$, and let $\delta=0$ or 1 according to whether $\bar{\rho}$ is finite or not at $p$. Consider a finite set of primes $Q=\left\{q_{2}, \cdots, q_{2 r}\right\}$ of odd cardinality (the reason for this eccentric numbering will be apparent) such that $\bar{\rho}$ is unramified at primes in $Q, q_{i}$ is not \pm 1 $\bmod p$ for $q_{i} \in Q$ and such that $\operatorname{tr}\left(\bar{\rho}\left(\right.\right.$ Frob $\left.\left._{q_{i}}\right)\right)= \pm\left(q_{i}+1\right)$ for $q_{i} \in Q$ with Frob $_{q_{i}}$ a (arithmetic) Frobenius element at $q_{i}$. For the subset of primes of even cardinality $\left\{q_{1}, \cdots, q_{2(r-s)}\right\}$ (for any $0 \leq s \leq r$ ) of $Q \cup\left\{q_{1}\right\}$, with $q_{1}=t$ a fixed prime dividing $N(\bar{\rho}) p^{\delta}(>1)$ that we choose once and for all, let $B_{q_{1}, \cdots, q_{2(r-s)}}$ be the indefinite quaternion algebra ramified exactly at the set $\left\{q_{1}, \cdots, q_{2(r-s)}\right\}$. For the congruence subgroup $\Gamma_{0}\left(N(\bar{\rho}) p^{\delta} q_{2(r-s)+1} \cdots q_{2 r} / t\right)$ of $B_{q_{1}, \cdots, q_{2(r-s)}}$ denote the Jacobian of the corresponding Shimura curve by $J^{q_{1}, \cdots, q_{2(r-s)}}$. This has an action of the Hecke operators $T_{r}$ for primes $r$ coprime to the primes in $Q$ and coprime to $N(\bar{\rho}) p$. By virtue of the main theorem of $[\mathrm{DT}]$ and the Jacquet-Langlands correspondence there is a maximal ideal $\mathrm{m}$ of the $W(k)$-algebra generated by the induced action of these $T_{r}$ 's on $\operatorname{Ta}_{p}\left(J^{\left.q_{1}, \cdots, q_{2(r-s)}\right)} \otimes_{\mathbf{z}_{p}} W(k)\right.$ (where $\operatorname{Ta}_{p}\left(J^{q_{1}, \cdots, q_{2(r-s)}}\right)$ is as usual the $p$-adic Tate module of $J^{\left.q_{1}, \cdots, q_{2(r-s)}\right)}$ that gives rise to the representation $\bar{\rho}$, i.e., $T_{r}-\operatorname{tr}\left(\widehat{\bar{\rho}\left(\mathrm{Frob}_{r}\right)}\right) \in \mathrm{m}$ for $\operatorname{tr}\left(\widehat{\bar{\rho}\left(\mathrm{Frob}_{r}\right)}\right)$ an arbitrary lift to $W(k)$ of $\operatorname{tr}\left(\bar{\rho}\left(\right.\right.$ Frob $\left.\left._{r}\right)\right)$ and Frob $_{r}$ a (arithmetic) Frobenius element at $r$. We denote by $\mathbf{T}_{Q}^{\left\{q_{1}, \cdots, q_{2(r-s)}\right\}-\text { new }}$ the completion of this Hecke algebra at $\mathbf{m}$ : it is finite flat as a $W(k)$ module. 
To define the minimal Hecke ring $\mathbf{T}_{\phi}$, we consider $\operatorname{Ta}_{p}\left(J_{0}\left(N(\bar{\rho}) p^{\delta}\right)\right) \otimes_{\mathbf{z}_{p}}$ $W(k)$ where $\operatorname{Ta}_{p}\left(J_{0}\left(N(\bar{\rho}) p^{\delta}\right)\right)$ is the $p$-adic Tate module of the Jacobian $J_{0}\left(N(\bar{\rho}) p^{\delta}\right)$ of the modular curve $X_{0}\left(N(\bar{\rho}) p^{\delta}\right)$ : this has an action of the Hecke operators $T_{r}$ for primes $r$ that are coprime to $N(\bar{\rho}) p$ and the primes in the chosen finite set of primes $Q$. The $W(k)$-algebra generated by the images of these $T_{r}$ 's in the $W(k)$-endomorphisms of $\operatorname{Ta}_{p}\left(J_{0}\left(N(\bar{\rho}) p^{\delta}\right)\right) \otimes \mathbf{z}_{p} W(k)$ is finite and flat over $W(k)$. By results of [Ri] (see [Ri1] for a proof that does not invoke multiplicity one results) there is a maximal ideal $\mathrm{m}$ of this Hecke algebra that gives rise to $\bar{\rho}$ in the sense explained above. We denote by $\mathbf{T}_{\phi}$ the completion at $\mathrm{m}$ of this Hecke algebra.

Lemma 1 We have representations $\rho_{Q, \text { mod }}^{\left\{q_{1}, \cdots, q_{2(r-s)}\right\}-\text { new }}: G_{\mathbf{Q}} \rightarrow G L_{2}\left(\mathbf{T}_{Q}^{\left\{q_{1}, \cdots, q_{2(r-s)}\right\}-\text { new }}\right)$ (resp., $\rho_{\phi, \text { mod }}: G_{\mathbf{Q}} \rightarrow G L_{2}\left(\mathbf{T}_{\phi}\right)$ ) that are unramified outside primes in $S \cup Q$ and the infinite place, and are characterised by $\operatorname{tr}\left(\rho_{Q, \bmod }^{\left\{q_{1}, \cdots, q_{2(r-s)}\right\}-n e w}\left(\right.\right.$ Frob $\left.\left._{r}\right)\right)=$ $T_{r}$ (resp., $\operatorname{tr}\left(\rho_{\phi, \text { mod }}\left(\right.\right.$ Frob $\left.\left.\left._{r}\right)\right)=T_{r}\right)$, that arise by uniquely determined specialisations of the universal representations $\rho_{Q}^{\left\{q_{2}, \cdots, q_{2(r-s)}\right\}-\text { new }}: G_{\mathbf{Q}} \rightarrow G L_{2}\left(R_{Q}^{\left\{q_{2}, \cdots, q_{2(r-s)}\right\}-\text { new }}\right)$ (resp., $\rho_{\phi}: G_{\mathbf{Q}} \rightarrow G L_{2}\left(R_{\phi}\right)$ ). The aforementioned specialisation maps $R_{Q}^{\left\{q_{2}, \cdots, q_{2(r-s)}\right\}-\text { new }} \rightarrow \mathbf{T}_{Q}^{\left\{q_{1}, \cdots, q_{2(r-s)}\right\} \text {-new }}$ (resp., $R_{\phi} \rightarrow \mathbf{T}_{\phi}$ ) of local $W(k)$ algebras are surjective.

Proof: The existence of the representations $\rho_{Q, \text { mod }}^{\left\{q_{1}, \cdots, q_{2(r-s)}\right\}-\text { new }}$ and $\rho_{\phi, \text { mod }}$ with the asserted properties follows from the results of $[\mathrm{Ca}]$ and the rest from the universal properties of deformation rings and the properties of the representations $\rho_{Q, \text { mod }}^{\left\{q_{1}, \cdots, q_{2(r-s)}\right\}-\text { new }}$ and $\rho_{\phi, \text { mod }}$ deduced from [Ca1]: for instance $\left.\rho_{Q, \text { mod }}^{\left\{q_{1}, \cdots, q_{2(r-s)}\right\}-\text { new }}\right|_{G_{q}}$ is of the form

$$
\pm\left(\begin{array}{cc}
\varepsilon & * \\
0 & 1
\end{array}\right)
$$

by $[\mathrm{Ca} 1]$.

\subsection{Isomorphisms between deformation rings and Hecke rings}

Definition 2 For $\rho: G_{\mathbf{Q}} \rightarrow G L_{2}(W(k))$ or $\rho: G_{\mathbf{Q}} \rightarrow G L_{2}\left(W(k) / p^{n}\right)$ we say that $\rho$ is special at a prime $q$ if $q$ is not congruent to $\pm 1 \bmod p$, and

$$
\left.\rho\right|_{G_{q}} \simeq \pm\left(\begin{array}{cc}
\varepsilon & * \\
0 & 1
\end{array}\right) .
$$


The following theorem and its proof we owe to Ravi Ramakrishna: we use the notation of Sections 2.1 and 2.2.

Theorem 1 There exists a finite set of primes $Q=\left\{q_{2}, \cdots, q_{2 r}\right\}$ of odd cardinality for some integer $r$ such that for $q \in Q, \bar{\rho}$ is unramified and special at $q$ and we have the sequence of isomorphisms $R_{Q}^{Q-\text { new }} \simeq \mathbf{T}_{Q}^{Q-\text { new }} \simeq W(k)$ of local $W(k)$-algebras.

Proof. Using Proposition 21 of $[\mathrm{KR}]$ we get a finite set of primes $Q=$ $\left\{q_{2}, \cdots, q_{2 r}\right\}$ such that for $q \in Q, q$ is special for $\bar{\rho}$ and such that the map

$$
H^{1}\left(G_{S \cup Q}, \operatorname{Ad}^{0}(\bar{\rho})\right) \rightarrow \oplus_{v \in S \cup Q} H^{1}\left(G_{v}, \operatorname{Ad}^{0}(\bar{\rho})\right) / \mathcal{N}_{v}
$$

is an isomorphism. Thus by Proposition $1, R_{Q}^{Q-n e w}$ is a quotient of $W(k)$. The main theorem of [DT] yields a $p$-adic modular lift of $\bar{\rho}$, that arises from a specialisation of the universal representation $\rho_{Q}^{Q-\text { new }}: G_{\mathbf{Q}} \rightarrow G L_{2}\left(R_{Q}^{Q-\text { new }}\right)$. As by Lemma 1 we a priori do have a surjection $R_{Q}^{Q-\text { new }} \rightarrow \mathbf{T}_{Q}^{Q-\text { new }}$ the isomorphisms in the statement follow.

Definition 3 A finite set of primes $Q$ such that $R_{Q}^{Q-\text { new }} \simeq W(k)$ as in the theorem is called an auxiliary set and the corresponding representation $G_{\mathbf{Q}} \rightarrow$ $G L_{2}(W(k))$ is denoted by $\rho_{Q}^{Q-\text { new }}$.

A new proof of the following theorem of [W] and [TW], that will deduce it from Theorem 1, will be given in the next section and is the main contribution of this article.

Theorem 2 We have an isomorphism $R_{Q} \simeq \mathbf{T}_{Q}$ of complete intersection rings.

We have the following corollary proved in [W] and [TW] that is deduced from Theorem 2 using the methods of Section 2 of [W], i.e., level raising methods as in [Ri2] and [DT] and the numerical isomorphism criterion of Wiles.

Corollary 1 Let $\rho: G_{\mathbf{Q}} \rightarrow G L_{2}(\mathcal{O})$ be a lift of $\bar{\rho}$, with $\mathcal{O}$ the ring of integers of a finite extension of $\mathbf{Q}_{p}$, such that $\rho$ is unramified at almost all places, with determinant the p-adic cyclotomic character $\varepsilon$, such that $\left.\rho\right|_{I_{p}}$ either arises from a p-divisible group scheme or $\left.\rho\right|_{I_{p}}$ is of the form

$$
\left(\begin{array}{ll}
\varepsilon & * \\
0 & 1
\end{array}\right)
$$

Then $\rho$ is modular. 
We also have the following corollary that can be deduced from Theorem 2: this deduction is in the appendix by Gebhard Böckle [B] to this paper.

Corollary $\mathbf{2}$ We have an isomorphism $R_{\phi} \simeq \mathbf{T}_{\phi}$ of complete intersection rings.

\section{Proof of Theorem $[2$}

\subsection{Some preliminaries}

We begin by stating 2 results needed below. The first is the vital commutative algebra result of [W] as refined by Lenstra (cf. Theorem 5.3 of [DDT]). (We say that a $W(k)$-algebra $R$ that is a complete Noetherian local ring, and is finite flat as a $W(k)$ module, with residue field $k$, is a complete intersection if $R \simeq W(k)\left[\left[T_{1}, \cdots, T_{n}\right]\right] /\left(f_{1}, \cdots, f_{n}\right)$ for some $n$.)

Theorem 3 Let $R$ and $T$ be complete Noetherian local $W(k)$-algebras with residue field $k, T$ finite flat as a $W(k)$ module, and $\phi: R \rightarrow T$ a surjective map of local $W(k)$-algebras. Let $\pi: T \rightarrow W(k)$ be a homomorphism of local $W(k)$-algebras, and set $\Phi_{R}=\operatorname{ker}(\pi \phi) / \operatorname{ker}(\pi \phi)^{2}$ and $\eta_{T}=\pi\left(\operatorname{Ann}_{T}(\operatorname{ker}(\pi))\right)$. Then $\left|W(k) / \eta_{T}\right| \leq\left|\Phi_{R}\right|$, where in the case $\left|W(k) / \eta_{T}\right|$ is infinite this is interpreted to mean that $\Phi_{R}$ is infinite too. Assume that $\eta_{T}$ is not zero. Then the following are equivalent:

- The equality $\left|\Phi_{R}\right|=\left|W(k) / \eta_{T}\right|$ is satisfied.

- The rings $R$ and $T$ are complete intersections, and $\phi$ is an isomorphism.

The second result we need is an upper bound on the orders of the tangent spaces at $\pi$ of the deformation rings $R_{Q}^{\alpha-\text { new }}$ where $\alpha$ is a subset of the finite set $Q, Q$ is as in Theorem 1 and $\pi$ is the map that is obtained by composing the natural map $R_{Q}^{\alpha-\text { new }} \rightarrow R_{Q}^{Q-\text { new }}$ with the isomorphism $R_{Q}^{Q-\text { new }} \simeq W(k)$ of Theorem 1. We define $\Phi_{\pi}\left(R_{Q}^{\alpha-n e w}\right)=\operatorname{ker}(\pi) / \operatorname{ker}(\pi)^{2}$. The representation $\rho_{Q}^{Q-n e w}: G_{\mathbf{Q}} \rightarrow G L_{2}(W(k))$ is residually absolutely irreducible and thus is integrally well-defined by [Ca]. Looking at the semistable representation

$\left.\rho_{Q}^{Q-\text { new }}\right|_{G_{q}}$ for any prime $q \in Q$ we have ideals $\left(x_{q}\right)$ of $W(k)$, where $\rho_{Q}^{Q-\text { new }}\left(t_{q}\right)$, with $t_{q}$ generating the unique $\mathbf{Z}_{p}$ quotient of $I_{q}$, is of the form

$$
\left(\begin{array}{cc}
1 & x_{q} \\
0 & 1
\end{array}\right)
$$


for some $x_{q} \in W(k)$ and $\left(x_{q}\right)$ does not depend on the choice of an integral model for $\rho_{Q}^{Q-\text { new }}$. We note that $x_{q} \neq 0\left(\right.$ as $R_{Q}^{Q-\text { new }} \simeq \mathbf{T}_{Q}^{Q-\text { new }}$ by Theorem 1 and then use [Ca1]). We have the following result that is proved in Section 5 of $[\mathrm{KR}]$ by computations of orders of some local Galois cohomology groups.

Proposition $2\left|\Phi_{\pi}\left(R_{Q}^{\alpha-n e w}\right)\right| \leq \Pi_{q \in Q \backslash \alpha}\left|W(k) /\left(x_{q}\right)\right|$.

The strategy of the proof of Theorem 2 is to deduce it from Theorem 1 using the above 2 results and the result of [RT] to compute change of $\eta$ invariants when we relax newness conditions on the deformation and Hecke rings two primes at a time.

We use the notation of Section 2 and consider an auxiliary set $Q=$ $\left\{q_{2}, \cdots, q_{2 r}\right\}$ provided by Theorem 11. Then the isomorphism $\pi: \mathbf{T}_{Q}^{Q-\text { new }} \rightarrow$ $W(k)$ of Theorem 1 induces a morphism $\mathbf{T}_{Q}^{\left\{q_{1}, \cdots, q_{2(r-s)}\right\}-\text { new }} \rightarrow W(k)$ of local $W(k)$-algebras using the Jacquet-Langlands correspondence: by an abuse of notation we again denote this morphism by $\pi$.

Let $\xi_{s}: J_{\Gamma}^{q_{1}, \cdots, q_{2 r-2 s}} \rightarrow A_{s}$ be the optimal quotient that corresponds to the morphism $\pi(0 \leq s \leq r-1)$ : thus $A_{s}$ is an abelian variety over $\mathbf{Q}$ such that $\rho_{Q}^{Q-\text { new }}$ arises from its $p$-adic Tate module, the map $\xi_{s}$ is equivariant for the Hecke action and its kernel is connected. Analogously we have the optimal quotient $\xi_{s}: J_{\Gamma}^{q_{1}, \cdots, q_{2 r-2(s+1)}} \rightarrow A_{s+1}$. (The optimal quotients that we consider are all isogenous to each other by Faltings' isogeny theorem, via Hecke-equivariant isogenies defined over Q.) We need to study the maps $\operatorname{Ta}_{p}\left(J^{q_{1}, \cdots, q_{2 r-2 s}}\right) \rightarrow \mathrm{Ta}_{p}\left(A_{s}\right)$ and $\operatorname{Ta}_{p}\left(J^{q_{1}, \cdots, q_{2 r-2(s+1)}}\right) \rightarrow \mathrm{Ta}_{p}\left(A_{s+1}\right)$ that $\xi_{s}$ and $\xi_{s+1}$ induce on the corresponding Tate modules. With the help of these we will be able to compare the $\eta$-invariant of the homomorphism $\pi: \mathbf{T}_{Q}^{\left\{q_{1}, \cdots, q_{2 r-2(s+1)}\right\}-\text { new }} \rightarrow W(k)$ (corresponding to $\xi_{s+1}$ ) to the $\eta$-invariant of the homomorphism $\pi: \mathbf{T}_{Q}^{\left\{q_{1}, \cdots, q_{2 r-2 s}\right\}-\text { new }} \rightarrow W(k)$ (corresponding to $\xi_{s}$ ). To ease the notation, we set $J:=J^{q_{1}, \cdots, q_{2 r-2 s}}$ and $J^{\prime}:=J^{q_{1}, \cdots, q_{2 r-2(s+1)}}, A_{s}:=A$ and $A_{s+1}:=A^{\prime}, \xi:=\xi_{s}$ and $\xi^{\prime}:=\xi_{s+1}$ and the corresponding Hecke algebras $\mathbf{T}_{Q}^{\left\{q_{1}, \cdots, q_{2 r-2 s}\right\}-\text { new }}$ and $\mathbf{T}_{Q}^{\left\{q_{1}, \cdots, q_{2(r-2(s+1))}\right\}-\text { new }}$ by $\mathbf{T}$ and $\mathbf{T}^{\prime}$ respectively. We also set $q_{2(r-s)}=q$ and $q_{2(r-s)-1}=q^{\prime}$.

We have induced maps $\xi^{\prime *}: \mathrm{Ta}_{p}\left(\left(A^{\prime}\right)^{d}\right)_{\mathrm{m}, W(k)} \rightarrow \mathrm{Ta}_{p}\left(J^{\prime}\right)_{\mathrm{m}, W(k)}$, and $\xi_{*}^{\prime}:$ $\operatorname{Ta}_{p}\left(J^{\prime}\right)_{\mathrm{m}, W(k)} \rightarrow \mathrm{Ta}_{p}\left(A^{\prime}\right)_{\mathrm{m}, W(k)}$ (injective with torsion-free cokernel and surjective respectively by optimality, and their analogs for $\xi$ ), where for instance $\mathrm{Ta}_{p}\left(\left(A^{\prime}\right)^{d}\right)_{\mathrm{m}, W(k)}:=\left(\operatorname{Ta}_{p}\left(\left(A^{\prime}\right)^{d}\right) \otimes_{\mathbf{z}_{p}} W(k)\right)_{\mathrm{m}}$ is the localisation at $\mathrm{m}$ of $p$-adic Tate module of the dual abelian variety $\left(A^{\prime}\right)^{d}:=\operatorname{Pic}^{0}\left(A^{\prime}\right)$ of $A^{\prime}$ tensored 
with $W(k)$, and the other symbols are defined analogously. (It's worth noting that if $k$ is the field of definition of $\bar{\rho}, \mathcal{O}$ is the image of the Hecke algebra in $\operatorname{End}\left(A^{\prime}\right)$ and $\lambda$ is the prime of $\mathcal{O}$ corresponding to $\mathrm{m}$, then $\mathcal{O}_{\lambda} \cong W(k)$, $\operatorname{Ta}_{p}\left(A^{\prime}\right)_{\mathrm{m}, W(k)} \cong \mathrm{Ta}_{p}\left(A^{\prime}\right)_{\lambda}$, etc.) Observe that $\operatorname{Ta}_{p}\left(\left(A^{\prime}\right)^{d}\right)_{\mathrm{m}, W(k)}$ is free of rank 2 over the quotient of $\mathbf{T}^{\prime}$ cut out by its action on it: this quotient is isomorphic to $W(k)$ (a section of the structure map $W(k) \rightarrow \mathbf{T}^{\prime}$ ). The module $\operatorname{Ta}_{p}\left(\left(A^{\prime}\right)^{d}\right)_{\mathrm{m}, W(k)}$ is (non-canonically) isomorphic to $\operatorname{Ta}_{p}\left(A^{\prime}\right)_{\mathrm{m}, W(k)}$ as a $W(k)\left[G_{\mathbf{Q}}\right]$ module by $[\mathrm{Ca}]$. We identify these by choosing any such isomorphism: the ambiguity is only up to elements of $W(k)^{*}$ which is immaterial in the calculations below. Analogously we have the maps induced by $\xi$ involving a choice of a $W(k)\left[G_{\mathbf{Q}}\right]$-isomorphism between localisations at $\mathrm{m}$ of the Tate modules of $A$ and its dual. The maps $\xi_{*}^{\prime} \xi^{*}$ and $\xi_{*} \xi^{*}$ of $\operatorname{Ta}_{p}\left(A^{\prime}\right)_{\mathrm{m}, W(k)}$ and $\operatorname{Ta}_{p}(A)_{\mathrm{m}, W(k)}$ commute with the $W(k)\left[G_{\mathbf{Q}}\right]$-action and by the irreducibility of this action can be regarded as given by multiplication by elements of $W(k)$. We denote the corresponding ideals of $W(k)$ by $\left(\xi_{*}^{\prime} \xi^{*}\right)$ and $\left(\xi_{*} \xi^{*}\right)$.

\subsection{A result of Ribet and Takahashi}

The following theorem which follows from an adaptation of the methods of [RT] to our situation is key to the deduction of Theorem 2 from Theorem 11.

Theorem $4\left(\xi_{*}^{\prime} \xi^{*}\right)=\left(x_{q} x_{q^{\prime}}\right)\left(\xi_{*} \xi^{*}\right)$ (where when $s=r-1$ we declare $x_{q^{\prime}}$ to be a unit).

Proof: Our argument is essentially that of Sections 2 and 3 of [RT], and below we just make the necessary adaptations of their arguments, that are in the case of optimal elliptic curve quotients of the above Jacobians, to make them go through in the case of optimal abelian variety quotients that we consider here which do not come equipped with canonical principal polarisations. The key point in [RT] as well as here is to consider character groups of the toric parts of the reductions of $J$ and $J^{\prime}$ at $q^{\prime}$ and $q$ respectively that are related by Ribet's exact sequence (2) below. The general facts about semistable abelian varieties that we use are found in $[\mathrm{G}]$.

Consider $\mathcal{X}\left(J^{\prime}, q\right)_{\mathrm{m}, W(k)}=\left(\mathcal{X}\left(J^{\prime}, q\right) \otimes_{\mathbf{Z}} W(k)\right)_{\mathrm{m}}$ and $\mathcal{X}\left(J, q^{\prime}\right)_{\mathrm{m}, W(k)}=\left(\mathcal{X}\left(J, q^{\prime}\right) \otimes_{\mathbf{Z}} W(k)\right)_{\mathrm{m}}$, where $\mathcal{X}\left(J^{\prime}, q\right)$ and $\mathcal{X}\left(J, q^{\prime}\right)$ are the character groups of the tori associated to the special fibre of Néron models of the abelian varieties $J^{\prime}$ and $J$, that are defined over $\mathbf{Q}$, at $q$ and $q^{\prime}$ respectively. These have functorial actions by the Hecke algebras $\mathbf{T}^{\prime}$ and $\mathbf{T}$ respectively. We analogously consider the 
character groups (tensored with $W(k)$ and localised at $\mathrm{m}) \mathcal{X}\left(A, q^{\prime}\right)_{\mathrm{m}, W(k)}$ and $\mathcal{X}\left(A^{\prime}, q\right)_{\mathrm{m}, W(k)}$ of the tori of the reduction of the Néron model of the abelian varieties $A, A^{\prime}$ at the primes $q^{\prime}$ and $q$ respectively. Note that as $A^{\prime}$ has purely toric reduction at $q$, we have the exact sequence of $W(k)\left[G_{q}\right]$-modules

$0 \rightarrow \operatorname{Hom}_{W(k)}\left(\mathcal{X}\left(A^{\prime}, q\right)_{\mathrm{m}, W(k)}, W(k)(1)\right) \rightarrow \mathrm{Ta}_{p}\left(A^{\prime}\right)_{\mathrm{m}, W(k)} \rightarrow \mathcal{X}\left(\left(A^{\prime}\right)^{d}, q\right)_{\mathrm{m}, W(k)} \rightarrow 0$

and the analogous exact sequence for the Tate module of the dual abelian variety $\left(A^{\prime}\right)^{d}$. Now we claim that the $W(k)\left[G_{\mathbf{Q}}\right]$-equivariant isomorphism between $\operatorname{Ta}_{p}\left(A^{\prime}\right)_{\mathrm{m}, W(k)}$ and $\mathrm{Ta}_{p}\left(\left(A^{\prime}\right)^{d}\right)_{\mathrm{m}, W(k)}$ chosen above induces yields a commutative diagram

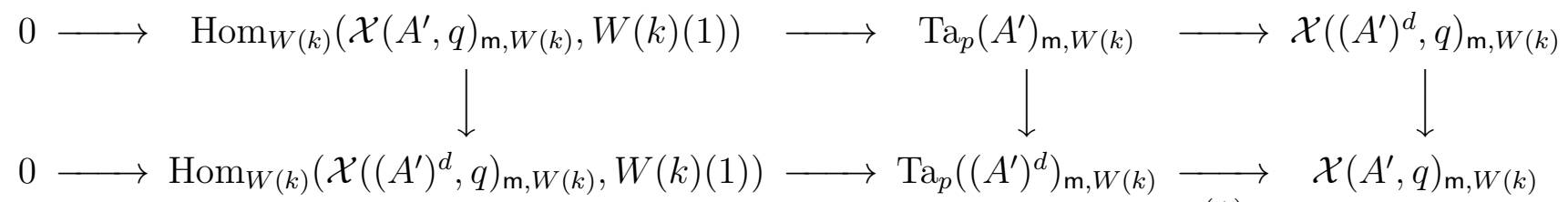

in which all the vertical arrows are ismorphisms. This follows from the fact that the middle vertical arrow being $W(k)\left[G_{q}\right]$-equivariant, it induces an isomorphism

$\operatorname{Hom}_{W(k)}\left(\mathcal{X}\left(A^{\prime}, q\right)_{\mathrm{m}, W(k)}, W(k)(1)\right) \otimes \mathbf{Q}_{p} \simeq \operatorname{Hom}_{W(k)}\left(\mathcal{X}\left(\left(A^{\prime}\right)^{d}, q\right)_{\mathrm{m}, W(k)}, W(k)(1)\right) \otimes \mathbf{Q}_{p}$.

Noting that the cokernels of the maps in the horizontal exact sequences above are torsion-free establishes the claim. (When $q \neq p$ this follows more simply from noting that the first term in each sequence is the module of $I_{q}$-invariants.)

We have an injective map $\xi_{X}^{* *}: \mathcal{X}\left(A^{\prime}, q\right)_{\mathrm{m}, W(k)} \rightarrow \mathcal{X}\left(J^{\prime}, q\right)_{\mathrm{m}, W(k)}$ induced by pull-back from $\xi^{\prime}$ and similarly a surjective map $\xi_{X, *}^{\prime}: \mathcal{X}\left(J^{\prime}, q\right)_{\mathrm{m}, W(k)} \rightarrow$ $\mathcal{X}\left(\left(A^{\prime}\right)^{d}, q\right)_{\mathrm{m}, W(k)}$ induced by pull-back from the map dual to $\xi^{\prime}$ from $\left(A^{\prime}\right)^{d} \rightarrow$ $J^{\prime}$. Note that as $J^{\prime}$ has semistable reduction at $q$, by results of $[\mathrm{G}]$ we have an inclusion $\operatorname{Hom}_{W(k)}\left(\mathcal{X}\left(J^{\prime}, q\right)_{\mathrm{m}, W(k)}, W(k)(1)\right) \rightarrow \operatorname{Ta}_{p}\left(J^{\prime}\right)_{\mathrm{m}, W(k)}$ that is $\mathbf{T}^{\prime}\left[G_{q}\right]$ equivariant. Further, the map $\xi^{\prime *}: \mathrm{Ta}_{p}\left(\left(A^{\prime}\right)^{d}\right)_{\mathrm{m}, W(k)} \rightarrow \mathrm{Ta}_{p}\left(J^{\prime}\right)_{\mathrm{m}, W(k)}$ (that is induced from the map $\left(A^{\prime}\right)^{d} \rightarrow J^{\prime}$ dual to $\xi^{\prime}$ as above) induces a Hecke equivariant map

$$
\operatorname{Hom}_{W(k)}\left(\mathcal{X}\left(\left(A^{\prime}\right)^{d}, q\right)_{\mathrm{m}, W(k)}, W(k)(1)\right) \rightarrow \operatorname{Hom}_{W(k)}\left(\mathcal{X}\left(J^{\prime}, q\right)_{\mathrm{m}, W(k)}, W(k)(1)\right)
$$

of $W(k)\left[G_{q}\right]$-modules that is $\operatorname{Hom}\left(\xi_{X, *}^{\prime}, W(k)(1)\right)$. These considerations show that the sequence of maps

$$
\operatorname{Ta}_{p}\left(A^{\prime}\right)_{\mathrm{m}, W(k)} \simeq \operatorname{Ta}_{p}\left(\left(A^{\prime}\right)^{d}\right)_{\mathrm{m}, W(k)} \rightarrow \operatorname{Ta}_{p}\left(J^{\prime}\right)_{\mathrm{m}, W(k)} \rightarrow \mathrm{Ta}_{p}\left(A^{\prime}\right)_{\mathrm{m}, W(k)},
$$


(where the first map is the isomorphism we have fixed) induces the sequence of maps

$$
\begin{aligned}
& \operatorname{Hom}_{W(k)}\left(\mathcal{X}\left(A^{\prime}, q\right)_{\mathrm{m}, W(k)}, W(k)(1)\right) \simeq \operatorname{Hom}_{W(k)}\left(\mathcal{X}\left(\left(A^{\prime}\right)^{d}, q\right)_{\mathrm{m}, W(k)}, W(k)(1)\right) \\
& \rightarrow \operatorname{Hom}_{W(k)}\left(\mathcal{X}\left(J^{\prime}, q\right)_{\mathrm{m}, W(k)}, W(k)(1)\right) \rightarrow \operatorname{Hom}_{W(k)}\left(\mathcal{X}\left(A^{\prime}, q\right)_{\mathrm{m}, W(k)}, W(k)(1)\right)
\end{aligned}
$$

(where for instance the first map is the isomorphism deduced from (11) and the last map is $\left.\operatorname{Hom}\left(\xi_{X}^{*}, W(k)(1)\right)\right)$. As the composite of the maps in the first sequence is multiplication by $\xi_{*}^{\prime} \xi^{\prime *} \in W(k)$, we deduce that the composite of the maps in the second sequence is also multiplication by $\xi_{*}^{\prime} \xi^{\prime *}$.

The isomorphism of $W(k)\left[G_{q}\right]$-modules $\operatorname{Hom}_{W(k)}\left(\mathcal{X}\left(\left(A^{\prime}\right)^{d}, q\right)_{\mathrm{m}, W(k)}, W(k)(1)\right) \simeq$ $\operatorname{Hom}_{W(k)}\left(\mathcal{X}\left(A^{\prime}, q\right)_{\mathrm{m}, W(k)}, W(k)(1)\right)$ induces an isomorphism $\mathcal{X}\left(A^{\prime}, q\right)_{\mathrm{m}, W(k)} \simeq$ $\mathcal{X}\left(\left(A^{\prime}\right)^{d}, q\right)_{\mathrm{m}, W(k)}$ which we use to identify these two latter modules. Using this isomorphism we will regard $\xi_{X, *}^{\prime}$ as a map $\xi_{X, *}^{\prime}: \mathcal{X}\left(J^{\prime}, q\right)_{\mathrm{m}, W(k)} \rightarrow$ $\mathcal{X}\left(A^{\prime}, q\right)_{\mathrm{m}, W(k)}$. From what we have said above it follows that the composition $\xi_{X, *}^{\prime} \xi_{X}^{\prime *}$ acts on the rank one $W(k)$-module $\mathcal{X}\left(A^{\prime}, q\right)_{\mathrm{m}, W(k)}$ by multiplication by an element of $W(k)$ which generates the ideal that we denoted by $\left(\xi_{*}^{\prime} \xi^{* *}\right)$ above. From this point onwards we will abuse notation and denote the maps $\xi_{X}^{*}$ and $\xi_{X, *}^{\prime}$ by $\xi^{* *}$ and $\xi_{*}^{\prime}$ respectively: by what has been said this is an acceptable abuse of notation. The same considerations apply to the map $\xi: J \rightarrow A$, and the induced maps on $p$-adic Tate modules (tensored with $W(k)$ and localised at $\mathrm{m}$ ) and the character groups of tori (tensored with $W(k)$ and localised at $\mathrm{m}$ ) associated to the special fiber at $q^{\prime}$ of the Néron models of $J$ and $A$.

We have the important Hecke equivariant exact sequence of Ribet

$$
0 \rightarrow \mathcal{X}\left(J, q^{\prime}\right)_{\mathrm{m}, W(k)} \rightarrow \mathcal{X}\left(J^{\prime}, q\right)_{\mathrm{m}, W(k)} \rightarrow \mathcal{X}\left(J^{\prime \prime}, q\right)_{\mathrm{m}, W(k)}^{2} \rightarrow 0
$$

where $\mathcal{X}\left(J^{\prime \prime}, q\right)_{\mathrm{m}, W(k)}$ is the character group of the torus associated to the fibre at $q$ (tensored with $W(k)$ and localised at $\mathrm{m}$ ) of the Néron model of the Jacobian of the Shimura curve arising from the congruence subgroup $\Gamma_{0}\left(N(\bar{\rho}) p^{\delta} q_{2(r-s)} \cdots q_{2 r} / t\right)$ of $B_{q_{1}, \cdots, q_{2(r-s)-2}}$. We will denote the map $\mathcal{X}\left(J, q^{\prime}\right)_{\mathrm{m}, W(k)} \rightarrow$ $\mathcal{X}\left(J^{\prime}, q\right)_{\mathrm{m}, W(k)}$ of the exact sequence (2) by $\iota$. The exact sequence (2) is deduced easily from Proposition 1 of [RT] (see also the original source, i.e., Theorem 4.1 of $[\mathrm{Ri}])$. Let us denote by the same symol $\mathcal{L}$ the submodules $\mathcal{X}(J)_{\mathrm{m}, W(k)}[\operatorname{ker}(\pi)]$ and $\mathcal{X}\left(J^{\prime}\right)_{\mathrm{m}, W(k)}[\operatorname{ker}(\pi)]$ (free of rank one over $W(k)$ ) of $\mathcal{X}(J)_{\mathrm{m}, W(k)}$ and $\mathcal{X}\left(J^{\prime}\right)_{\mathrm{m}, W(k)}$ respectively. This is an acceptable abuse of notation because the torsion-freeness of the cokernel of $\iota$, that follows from (2), implies that that these different $\mathcal{L}$ 's are identified by $\iota$. 
Recall the $W(k)$-bilinear monodromy pairings (see $[\mathrm{RT}]$ )

$$
\begin{aligned}
& (,)_{J}: \mathcal{X}\left(J, q^{\prime}\right)_{\mathrm{m}, W(k)} \times \mathcal{X}\left(J, q^{\prime}\right)_{\mathrm{m}, W(k)} \rightarrow W(k) \\
& (,)_{J^{\prime}}: \mathcal{X}\left(J^{\prime}, q\right)_{\mathrm{m}, W(k)} \times \mathcal{X}\left(J^{\prime}, q\right)_{\mathrm{m}, W(k)} \rightarrow W(k)
\end{aligned}
$$

for which the action of the operators $T_{r}$ is self-adjoint, and that are compatible with the injection

$$
\iota: \mathcal{X}\left(J, q^{\prime}\right)_{\mathrm{m}, W(k)} \rightarrow \mathcal{X}\left(J^{\prime}, q\right)_{\mathrm{m}, W(k)},
$$

of the exact sequence (2) above. We also have the $W(k)$-bilinear monodromy pairings

$$
\begin{aligned}
&(,)_{A^{\prime}}: \mathcal{X}\left(A^{\prime}, q\right)_{\mathrm{m}, W(k)} \times \mathcal{X}\left(A^{\prime}, q\right)_{\mathrm{m}, W(k)} \rightarrow W(k) \\
&(,)_{A}: \mathcal{X}\left(A, q^{\prime}\right)_{\mathrm{m}, W(k)} \times \mathcal{X}\left(A, q^{\prime}\right)_{\mathrm{m}, W(k)} \rightarrow W(k)
\end{aligned}
$$

where we are using the ismorphisms between $\mathcal{X}\left(A^{\prime}, q\right)_{\mathrm{m}, W(k)}$ and $\mathcal{X}\left(\left(A^{\prime}\right)^{d}, q\right)_{\mathrm{m}, W(k)}$ and $\mathcal{X}\left(A, q^{\prime}\right)_{\mathrm{m}, W(k)}$ and $\mathcal{X}\left((A)^{d}, q^{\prime}\right)_{\mathrm{m}, W(k)}$ discussed earlier. We denote the torsion cokernels of the corresponding maps into the duals, i.e., the component groups, by $\phi_{q^{\prime}}(J)_{\mathrm{m}, W(k)}, \phi_{q}\left(J^{\prime}\right)_{\mathrm{m}, W(k)}, \phi_{q^{\prime}}(A)_{\mathrm{m}, W(k)}$ and $\phi_{q}\left(A^{\prime}\right)_{\mathrm{m}, W(k)}$. (We can define the component groups at primes in $S$ analogously.) The maps $\xi$ and $\xi^{\prime}$ also induce maps $\xi_{*}: \phi_{q^{\prime}}(J)_{\mathrm{m}, W(k)} \rightarrow \phi_{q^{\prime}}(A)_{\mathrm{m}, W(k)}$ and $\xi_{*}^{\prime}: \phi_{q}\left(J^{\prime}\right)_{\mathrm{m}, W(k)} \rightarrow$ $\phi_{q}\left(A^{\prime}\right)_{\mathrm{m}, W(k)}$ that are considered in the following proposition.

Before stating the proposition note that by invoking the result of [Ca] we see that the $G L_{2}(W(k))$-valued representation of $G_{\mathbf{Q}}$ that arises from $\mathrm{Ta}_{p}(A)_{\mathrm{m}, W(k)} \simeq\left(\mathrm{Ta}_{p}(A) \otimes W(k)\right)_{\mathrm{m}}$ depends only on the (Hecke equivariant, Q-)isogeny class of $A$ and is isomorphic to $\rho_{Q}^{Q-\text { new }}\left(\simeq \operatorname{Ta}_{p}\left(A^{\prime}\right)_{\mathrm{m}, W(k)}\right)$.

Proposition 3 We have the following information about maps between component groups:

1. We have the equalities of orders of component groups: $\left|\phi_{q}(A)_{\mathrm{m}, W(k)}\right|=$ $\left|W(k) /\left(x_{q}\right)\right|=\left|\phi_{q}\left(A^{\prime}\right)_{\mathrm{m}, W(k)}\right|\left(x_{q}\right.$ was defined in 3.1) for any prime $q \in$ $Q$. Further, for any prime $\ell \mid N(\bar{\rho}) p^{\delta}$, the component groups $\phi_{\ell}\left(A^{\prime}\right)_{\mathrm{m}, W(k)}$ and $\phi_{\ell}(A)_{\mathrm{m}, W(k)}$ are trivial. The orders of these component groups depend only on the (Hecke equivariant, Q-)isogeny class of $A$ (or $\left.A^{\prime}\right)$.

2. The group $\phi_{q}\left(J^{\prime}\right)_{\mathrm{m}, W(k)}$ is trivial and hence the map $\xi_{*}^{\prime}: \phi_{q}\left(J^{\prime}\right)_{\mathrm{m}, W(k)} \rightarrow$ $\phi_{q}\left(A^{\prime}\right)_{\mathrm{m}, W(k)}$ is trivial.

3. The map $\xi_{*}: \phi_{q^{\prime}}(J)_{\mathrm{m}, W(k)} \rightarrow \phi_{q^{\prime}}(A)_{\mathrm{m}, W(k)}$ is surjective. 
Proof: The first part is well-known and follows easily from using nonarchimedean uniformisations of the abelian varieties $A$ and $A^{\prime}$ that have purely toric reduction at all $\ell \in Q$ and $\ell \mid N(\bar{\rho}) p^{\delta}$ (see Chapter III of [Ri3] and Section 3 of [GS] for instance). The referee has remarked that this also follows directly from the definition of the component group and the Néron mapping property. The point is that $\phi_{q}\left(A^{\prime}\right)\left[\lambda^{n}\right] \cong W(k) / p^{n}$ if and only if $A^{\prime}\left[\lambda^{n}\right]$ extends to a finite flat group scheme over $\mathbf{Z}_{q}$ (in the notation of the paragraph at the end of Section 3.1): see the proof of Lemma 6.2 of [Ri]. The last sentence of part 1 follows from all these considerations and the sentence before the statement of the proposition.

That $\phi_{q}\left(J^{\prime}\right)_{\mathrm{m}, W(k)}$ is trivial follows as $\mathrm{m}$ is non-Eisenstein (see Theorem 3.12 of [Ri] for an argument for this in a different situation but that works in ours too).

From part 1 it follows that the component group $\phi_{t}(A)_{\mathrm{m}, W(k)}$ is trivial. Thus part 3 follows from part 1 and from the methods of Section 3 of [RT] (see Proposition 3 and its corollary of loc. cit.) which show that the order of the cokernel of $\xi_{*}: \phi_{q^{\prime}}(J)_{\mathrm{m}, W(k)} \rightarrow \phi_{q^{\prime}}(A)_{\mathrm{m}, W(k)}$ divides the order of $\phi_{q_{1}}(A)_{\mathrm{m}, W(k)}=1$ (note that our chosen prime $t=q_{1}$ divides the discriminant of the the quaternion algebra from which $J$ arises for all values of $s$ $(0 \leq s \leq r-1)$ that we consider which makes the appeal to loc. cit. valid). This finishes the proof of the proposition.

Now arguing exactly as in Section 2 of [RT] we use the exact sequence (2) and the Proposition above to deduce Theorem 4. (Most parantheses below will indicate ideals of $W(k)$.) Consider $\mathcal{L}=\mathcal{X}\left(J^{\prime}, q\right)_{\mathrm{m}, W(k)}[\pi]$ : choose a generator $l$ of the $W(k)$-module $\mathcal{L}$ and set $(\tau):=\left((l, l)_{J^{\prime}}\right)$ where we are using the monodromy pairing recalled above. We have the maps as above $\xi^{* *}: \mathcal{X}\left(A^{\prime}, q\right)_{\mathrm{m}, W(k)} \rightarrow \mathcal{X}\left(J^{\prime}, q\right)_{\mathrm{m}, W(k)}$ and $\xi_{*}^{\prime}: \mathcal{X}\left(J^{\prime}, q\right)_{\mathrm{m}, W(k)} \rightarrow \mathcal{X}\left(A^{\prime}, q\right)_{\mathrm{m}, W(k)}$ (the second map results from the isomorphism chosen earlier between $\mathcal{X}\left(\left(A^{\prime}\right)^{d}, q\right)_{\mathrm{m}, W(k)}$ and $\left.\mathcal{X}\left(A^{\prime}, q\right)_{\mathrm{m}, W(k)}\right)$. Now by the adjointness property of $\xi^{\prime}$ with respect to the monodromy pairings above, i.e., $\left(\left(\xi^{*} x, y\right)_{J^{\prime}}\right)=\left(\left(x, \xi_{*}^{\prime} y\right)_{A^{\prime}}\right)$ for any $x \in \mathcal{X}\left(A^{\prime}, q\right)_{\mathrm{m}, W(k)}, y \in \mathcal{X}\left(J^{\prime}, q\right)_{\mathrm{m}, W(k)}$ (see discussion before Proposition 2 of $[\mathrm{RT}])$ we have that $\left(\xi_{*}^{\prime} \xi^{\prime *}\right)\left((x, x)_{A^{\prime}}\right)=\left(\left(\xi^{\prime *} x, \xi^{\prime *} x\right)_{J^{\prime}}\right)$. For a generator $x$ of the free $W(k)$ rank-one module $\mathcal{X}\left(A^{\prime}, q\right)_{\mathrm{m}, W(k)}$, we have that $\left((x, x)_{A^{\prime}}\right)=\left(x_{q}\right)$ from Proposition 3, and thus we deduce that

$$
\left(\xi_{*}^{\prime} \xi^{\prime *}\right)\left(x_{q}\right)=\left(\left[\mathcal{L}: \mathcal{X}\left(A^{\prime}, q\right)_{\mathrm{m}, W(k)}\right]^{1 / d}\right)^{2} \cdot(\tau)
$$


where $d$ is the rank of $W(k)$ as a $\mathbf{Z}_{p}$-module, and where we view $\mathcal{X}\left(A^{\prime}, q\right)_{\mathrm{m}, W(k)}$ as embedded in $\mathcal{L}$ by $\xi^{\prime *}$. Arguing similarly with respect to the map $\xi: J \rightarrow A$ and this time using the character groups of the tori of the reduction of $J$ and $A \bmod q^{\prime}$ and the equality $\left(\left(\iota^{-1}(l), \iota^{-1}(l)\right)_{J}\right)=(\tau)$, we get the analogous equality

$$
\left(\xi_{*} \xi^{*}\right)\left(x_{q^{\prime}}\right)=\left(\left[\mathcal{L}: \mathcal{X}\left(A, q^{\prime}\right)_{\mathrm{m}, W(k)}\right]^{1 / d}\right)^{2} \cdot(\tau) .
$$

Eliminating $\tau$ from these equalities we get the equation of fractional ideals of $W(k)$,

$$
\frac{\left(\xi_{*}^{\prime} \xi^{\prime *}\right)\left(x_{q}\right)}{\left(\left[\mathcal{L}: \mathcal{X}\left(A^{\prime}, q\right)_{\mathrm{m}, W(k)}\right]^{1 / d}\right)^{2}}=\frac{\left(\xi_{*} \xi^{*}\right)\left(x_{q^{\prime}}\right)}{\left(\left[\mathcal{L}: \mathcal{X}\left(A, q^{\prime}\right)_{\mathrm{m}, W(k)}\right]^{1 / d}\right)^{2}}
$$

Theorem 4 will follow from this and Proposition 3 once we have proved the following lemma (see Proposition 2 of $[\mathrm{RT}]$ ).

Lemma 2 The index $\left[\mathcal{L}: \mathcal{X}\left(A^{\prime}, q\right)_{\mathrm{m}, W(k)}\right]$ is the cardinality of the cokernel of $\xi_{*}^{\prime}: \phi_{q}\left(J^{\prime}\right)_{\mathrm{m}, W(k)} \rightarrow \phi_{q}\left(A^{\prime}\right)_{\mathrm{m}, W(k)}$ and the index $\left[\mathcal{L}: \mathcal{X}\left(A, q^{\prime}\right)_{\mathrm{m}, W(k)}\right]$ is the cardinality of the cokernel of $\xi_{*}: \phi_{q^{\prime}}(J)_{\mathrm{m}, W(k)} \rightarrow \phi_{q^{\prime}}(A)_{\mathrm{m}, W(k)}$.

Proof: The optimality of $\xi^{\prime}: J^{\prime} \rightarrow A^{\prime}$ implies that the dual map $\left(A^{\prime}\right)^{d} \rightarrow J^{\prime}$ is injective and thus the map of character groups $\xi_{*}^{\prime}: \mathcal{X}\left(J^{\prime}, q\right)_{\mathrm{m}, W(k)} \rightarrow$ $\mathcal{X}\left(\left(A^{\prime}\right)^{d}, q\right)_{\mathrm{m}, W(k)}$ is surjective. We use the isomorphism between $\mathcal{X}\left(A^{\prime}, q\right)_{\mathrm{m}, W(k)}$ and $\mathcal{X}\left(\left(A^{\prime}\right)^{d}, q\right)_{\mathrm{m}, W(k)}$ to regard $\xi_{*}^{\prime}$ as before as a map $\xi_{*}^{\prime}: \mathcal{X}\left(J^{\prime}, q\right)_{\mathrm{m}, W(k)} \rightarrow$ $\mathcal{X}\left(A^{\prime}, q\right)_{\mathrm{m}, W(k)}$. Again using this isomorphism and the prior description of the monodromy pairings we have the commutative diagram:

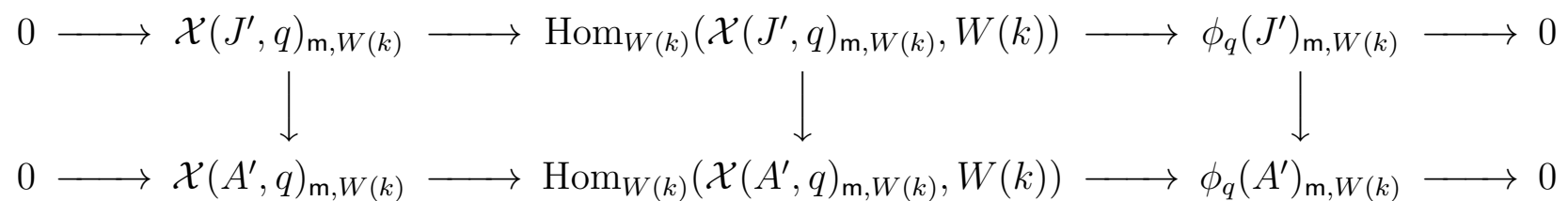

The first vertical map (which is the $\xi_{*}^{\prime}$ above) being surjective, the cokernels of $\operatorname{Hom}_{W(k)}\left(\xi^{* *}, W(k)\right)$ (which is the middle vertical map) and the right hand vertical map (which is the map $\xi_{*}^{\prime}: \phi_{q}\left(J^{\prime}\right)_{\mathrm{m}, W(k)} \rightarrow \phi_{q}\left(A^{\prime}\right)_{\mathrm{m}, W(k)}$ we are interested in) may be identified. But now the order of the cokernel of $\operatorname{Hom}_{W(k)}\left(\xi^{*}, W(k)\right)$ coincides with the order of the torsion subgroup of the cokernel of the map $\xi^{*}: \mathcal{X}\left(A^{\prime}, q\right)_{\mathrm{m}, W(k)} \rightarrow \mathcal{X}\left(J^{\prime}, q\right)_{\mathrm{m}, W(k)}$. But since $\mathcal{X}\left(J^{\prime}, q\right)_{\mathrm{m}, W(k)} / \mathcal{L}$ is torsion-free we derive the equality of the index 
$\left[\mathcal{L}: \mathcal{X}\left(A^{\prime}, q\right)_{\mathrm{m}, W(k)}\right]$ with the cardinality of the cokernel of $\xi_{*}^{\prime}: \phi_{q}\left(J^{\prime}\right)_{\mathrm{m}, W(k)} \rightarrow$ $\phi_{q}\left(A^{\prime}\right)_{\mathrm{m}, W(k)}$ as desired. The second statement of the lemma is proved entirely analogously and this finishes the proof of the lemma.

\subsection{End of proof}

We start with the isomorphism $R_{Q}^{Q-\text { new }} \simeq \mathbf{T}_{Q}^{Q \text {-new }}$ of Theorem 1. We apply Theorem 4 inductively to get the containment $\left(\xi_{r, *} \xi_{r}^{*}\right) \subset\left(\prod_{q \in Q} x_{q}\right)$ of ideals of $W(k)$. Consider the monodoromy pairing on $X=\mathcal{X}\left(J_{r}, t\right)_{\mathrm{m}, W(k)}$ that was considered earlier where $J_{r}=J_{0}\left(N(\bar{\rho}) p^{\delta} q_{2} \cdots q_{2 r}\right)$ : this is perfect as $\phi_{t}\left(J_{r}\right)_{\mathrm{m}, W(k)}=0$ (see part 2 of Proposition 3). By Lemma 2 and part 1 of Prop. 3, $\xi_{r}^{*}$ defines a map from $\mathcal{X}\left(A_{r}, t\right)_{\mathrm{m}, W(k)}$ to $X$ with torsion-free cokernel. Thus $\xi_{r}^{*}\left(\mathcal{X}\left(A_{r}, t\right)_{\mathrm{m}, W(k)}\right)=\mathcal{L}:=X[\operatorname{ker}(\pi)]$. Picking a generator $x$ of the free rank one $W(k)$-module $\mathcal{X}\left(A_{r}, t\right)_{\mathrm{m}, W(k)}$, we see that $\xi_{r}^{*}(x)$ generates $\mathcal{L}$, and that $\left(\left(\xi_{r}^{*}(x), \xi_{r}^{*}(x)\right)\right)=\left(\left(\xi_{r, *} \xi_{r}^{*}\right)(x, x)\right)=\left(\left(\xi_{r, *} \xi_{r}^{*}\right)\right)$ (equality of ideals of $W(k))$ where the pairings are the monodromy pairings on $X$ and $\mathcal{X}\left(A_{r}, t\right)_{\mathbf{m}, W(k)}$ respectively. The last equality follows from the fact that the component group $\phi_{t}\left(A_{r}\right)_{\mathrm{m}, W(k)}$ is trivial by part 1 of Proposition 3 . Now just as in proof of Lemma 4.17 of $[\mathrm{DDT}]$ we know that $X /(X[\operatorname{ker}(\pi)]+X[I])$ is a $W(k) / \pi(I)$-module (where $I=\mathrm{Ann}_{\mathbf{T}_{Q}}(\operatorname{ker}(\pi))$ ) isomorphic to the cokernel of $\mathcal{L} \rightarrow \operatorname{Hom}_{W(k)}(\mathcal{L}, W(k))$ (the map being induced by the monodromy pairing on $X)$ since $X / X[I] \cong \operatorname{Hom}_{W(k)}(X[\operatorname{ker}(\pi)], W(k))$. We conclude that $\left(\pi\left(\operatorname{Ann}_{\mathbf{T}_{Q}}(\operatorname{ker}(\pi))\right)\right) \subset\left(\prod_{q \in Q} x_{q}\right)$ and thus by Proposition 2 and Theorem 3 we deduce the isomorphism $R_{Q} \simeq \mathbf{T}_{Q}$.

\section{Remarks.}

- The presentation in 3.3 above using pairings on character groups was suggested by the referee. We had earlier used pairings on Tate modules. But note that to prove all the intermediate isomorphisms $R_{Q}^{Q_{s}-\text { new }} \rightarrow$ $\mathbf{T}_{Q}^{Q_{s}-\text { new }}$ (with $Q_{s}=\left\{q_{1}, \cdots, q_{2(r-s)}\right\}$ ) from the methods here one would in 3.3 above have to use pairings on Tate module of $A_{s}$. The pairings on character groups would not serve the purpose as the monodromy pairings would not be perfect (they would also not have faithful actions of the Hecke algebras that would be relevant unless $s=r$ ). On the other hand all these intermediate isomorphisms can be deduced from $R_{Q} \simeq \mathbf{T}_{Q}$ using the methods of the appendix [B]. 
- Note that in the proof of Theorem 2 we did not use any a priori multiplicity one results or Gorenstein properties of the Hecke algebras considered here. In $[\mathrm{D}]$ the construction in $[\mathrm{TW}]$ too was made independent of this input.

- In the proof the assumption $p>5$ is needed only as far as this is needed in [R1]. It is quite likely that the method of proof of Theorem 2 here be extended to $p>2$ (using the refinements of [R1] in [T]).

- Richard Taylor had sketched to the author a monodromy argument for computing change of $\eta$-invariants when one drops one prime at a time that was very helpful in showing the way towards the proof given above.

\section{$4 \quad$ Level substitution $\bmod p^{n}$}

We briefly sketch in this section another approach to lifting an isomorphism between new quotients of deformation and Hecke rings that works under the assumption that $N(\bar{\rho}) p^{\delta}$ is divisible by at least 2 primes (required for D. Helm's result in the appendix $[\mathrm{H}]$ ). The raison d'etre of this section is to indicate a method of "level substitution $\bmod p^{n}$ " (see Proposition (4) and we will skip details that can be filled in by standard arguments.

For any finite set of primes $Q=\left\{q_{1}, \cdots, q_{n}\right\}$ that are special for $\bar{\rho}$ (see Definition 2) and a subset $\alpha$ of $Q$ consider $H^{1}\left(X_{0}\left(N(\bar{\rho}) p^{\delta} Q\right), W(k)\right)^{\alpha-\text { new }}$ which is defined as the maximal torsion-free quotient of the quotient of $H^{1}\left(X_{0}\left(N(\bar{\rho}) p^{\delta} Q\right), W(k)\right)$ by the $W(k)$-submodule spanned by the images of $H^{1}\left(X_{0}\left(N(\bar{\rho}) p^{\delta} \frac{Q}{q}\right), W(k)\right)^{2}$ in $H^{1}\left(X_{0}\left(N(\bar{\rho}) p^{\delta} Q\right), W(k)\right)$, as $q$ runs through the primes of $\alpha$, under the standard degeneracy maps (here and below for a finite set of primes $Q$ we abusively denote by $Q$ again the product of the primes in it). We consider the standard action of Hecke operators $T_{r}$ for all primes $T_{r}$ (note that we are using $T_{r}$ for operators that sometimes get called $U_{r}$ to be consistent with Helm's appendix). By [DT] there is a maximal ideal $\mathrm{m}$ of the $W(k)$-algebra generated by the action of these $T_{r}$ 's such that $T_{r}-\operatorname{tr}\left(\widetilde{\bar{\rho}\left(\text { Frob }_{r}\right)}\right) \in \mathrm{m}$ for $\operatorname{tr}\left(\widetilde{\left.\bar{\rho}\left(\text { Frob }_{r}\right)\right)}\right)$ an arbitrary lift to $W(k)$ of $\operatorname{tr}\left(\bar{\rho}\left(\right.\right.$ Frob $\left.\left._{r}\right)\right)$ and $(r, N(\bar{\rho}) p Q)=1$, and $T_{r}-\tilde{\alpha_{r}} \in \mathrm{m}$ for $r \mid N(\bar{\rho}) p Q$, where $\alpha_{r}$ is the unique root of the characteristic polynomial of $\bar{\rho}\left(\mathrm{Frob}_{r}\right)$ congruent to \pm 1 when $r \mid Q$, it is the scalar by which (the arithmetic Frobenius) Frob ${ }_{r}$ acts 
on the unramified quotient of $\left.\bar{\rho}\right|_{G_{r}}$ when $r \mid N(\bar{\rho})$ or $r=p$ is ordinary for $\bar{\rho}$ (if $r=p$ and $\bar{\rho}$ is not ordinary at $p$ we take $\alpha_{r}$ to be 0 ), and $\tilde{\alpha_{r}}$ is any lift of $\alpha_{r}$ to $W(k)$. Then we define $\mathbf{T}_{Q}^{\alpha-\text { new }}$ to be the localisation at $\mathbf{m}$ of the $W(k)$ algebra generated by the action of these Hecke operators on the finite flat $W(k)$-module $H^{1}\left(X_{0}\left(N(\bar{\rho}) p^{\delta} Q\right), W(k)\right)^{\alpha-n e w}$. An analog of Lemma 1 gives that we have natural surjective maps $R_{Q}^{\alpha-\text { new }} \rightarrow \mathbf{T}_{Q}^{\alpha-\text { new }}$ (where we take care of the fact that all $T_{r}$ 's are in the image, including $r \mid N(\bar{\rho}) p^{\delta} Q$, as in Section 2 of [W] relying on results of [Ca1]).

We need properties of $\mathbf{T}_{Q}^{\alpha-\text { new }}$ that are proved $([\mathrm{H}]$ and Corollary 3$)$ by exploiting another description of these algebras that we recall for orienting the reader (although we do not make explicit use of the alternative descriptions in this sketch). For this fix a subset $\beta$ of the prime divisors of $N(\bar{\rho}) p^{\delta}$. Denote by $B_{\alpha, \beta}$ the quaternion algebra over $\mathbf{Q}$ ramified at the primes in $\alpha \cup \beta$ and further at $\infty$ if the cardinality $n^{\prime}=|\alpha \cup \beta|$ is odd. Denote by $\mathbf{A}$ the adeles over Q. For the standard open compact subgroup $U_{\alpha, \beta}:=U_{0}\left(N(\bar{\rho}) p^{\delta} Q \alpha^{-1} \beta^{-1}\right)$ of the $\mathbf{A}$-valued points of the algebraic group $G_{\alpha, \beta}$ (over $\mathbf{Q}$ ) corresponding to $B_{\alpha, \beta}, G_{\alpha, \beta}(\mathbf{A})$, we consider the coset space $\mathcal{X}_{U_{\alpha, \beta}}=G_{\alpha, \beta}(\mathbf{Q}) \backslash G_{\alpha, \beta}(\mathbf{A}) / U_{\alpha, \beta}$. Depending on whether $n^{\prime}$ is odd or even, this double coset space either is merely a finite set of points, or can be given the structure of a Riemann surface (that is compact if $n^{\prime} \neq 0$ and can be compactified by adding finitely many points if $n^{\prime}$ is 0 ). If $n^{\prime}$ is odd we consider the space of functions $\mathcal{S}_{U_{\alpha, \beta}}:=$ $\left\{f: \mathcal{X}_{U_{\alpha, \beta}} \rightarrow W(k)\right\}$ modulo the functions which factorise through the norm map, and in the case of $n^{\prime}$ even we consider the first cohomology of the corresponding Riemann surface $\mathcal{X}_{U_{\alpha, \beta}}$, i.e., $\mathcal{S}_{U_{\alpha, \beta}}:=H^{1}\left(\mathcal{X}_{U_{\alpha, \beta}}, W(k)\right)$. These $W(k)$-modules have the standard action of Hecke operators $T_{r}$ (see Sections 2 and 3 of [DT1]). By the results of [DT] and the Jacquet-Langlands correspondence there is a maximal ideal that we denote by $\mathrm{m}$ again in the support of the $W(k)$-algebra generated by the action of the $T_{r}$ 's on $\mathcal{S}_{U_{\alpha, \beta}}$ characterised as before. We denote the localisation at $\mathrm{m}$ of this Hecke algebra by $\mathbf{T}_{Q}^{\prime \alpha \cup \beta \text {-new }}$. Then by the Jacquet-Langlands correspondence, which gives an isomorphism $\mathbf{T}_{Q}^{\alpha \cup \beta \text {-new }} \otimes \mathbf{Q}_{p} \simeq \mathbf{T}_{Q}^{\alpha-\text { new }} \otimes \mathbf{Q}_{p}$ that takes $T_{r}$ to $T_{r}$, and the freeness of $\mathbf{T}_{Q}^{\alpha \cup \beta \text {-new }}$ and $\mathbf{T}_{Q}^{\alpha-\text { new }}$ as $W(k)$-modules, we have $\mathbf{T}_{Q}^{\alpha \cup \beta \text {-new }} \simeq \mathbf{T}_{Q}^{\alpha-\text { new }}$, an isomorphism of local $W(k)$-algebras.

We consider an auxiliary set $Q=\left\{q_{1}, \cdots, q_{n}\right\}$ as in Theorem 1 such that $R_{Q}^{Q-\text { new }} \simeq \mathbf{T}_{Q}^{Q-\text { new }} \simeq W(k)$, denote by $\pi^{\prime}$ all the asserted isomorphisms, and define the ideal $\left(x_{q_{i}}\right)$ of $W(k)$ as before in Section 3.1. We may assume that $Q \backslash\left\{q_{i}\right\}$ is not an auxiliary set (otherwise work with $Q \backslash\left\{q_{i}\right\}$ ). Then by the 
methods of $[R],[R 1],[T]$ and $[K R]$ we have the following proposition.

Proposition 4 For each $q_{i} \in Q$ such that $x_{q_{i}} \in p^{m_{i}} W(k)-p^{m_{i}+1} W(k)$ there is a prime $q_{i}^{\prime}$ not in $S \cup Q$ which is special for $\rho_{Q}^{Q-\text { new }} \bmod p^{m_{i}}$ and such that $Q_{i}=Q \backslash\left\{q_{i}\right\} \cup\left\{q_{i}^{\prime}\right\}$ is an auxiliary set. Hence we have isomorphisms $R_{Q_{i}}^{Q_{i}-\text { new }} \simeq \mathbf{T}_{Q_{i}}^{Q_{i}-\text { new }} \simeq W(k)$ and further $\rho_{Q}^{Q-\text { new }} \simeq \rho_{Q_{i}}^{Q_{i}-\text { new }} \bmod p^{m_{i}}$.

Proof: We use Lemma 8 of the companion paper [KR]. As $Q \backslash\left\{q_{i}\right\}$ is not an auxiliary set, it follows using the notation of loc. cit. that $H_{\mathcal{N}_{v}}^{1}\left(G_{S \cup Q_{i}}, \operatorname{Ad}^{0}(\bar{\rho})\right)$ and $H_{\mathcal{N}_{v}^{\perp}}^{1}\left(G_{S \cup Q_{i}}, \operatorname{Ad}^{0}(\bar{\rho})(1)\right)$ are 1-dimensional spanned by $\psi$ and $\phi$ respectively. Then using loc. cit. we choose $q_{i}^{\prime}$ not in $S \cup Q$ such that $\left.\psi\right|_{G_{q_{i}^{\prime}}} \neq 0$, $\left.\phi\right|_{G_{q_{i}^{\prime}}} \neq 0$, and $\rho_{Q}^{Q-\text { new }} \bmod p^{m_{i}}$ is special at $q_{i}^{\prime}$. This is the $q_{i}^{\prime}$ that we want.

Set $Q^{\prime}=\left\{q_{1}^{\prime}, \cdots, q_{n}^{\prime}\right\}$. From the result of $[\mathrm{H}]$ and the isomorphisms $\mathbf{T}_{Q}^{\alpha \cup \beta-\text { new }} \simeq \mathbf{T}_{Q}^{\alpha-\text { new }}$ it follows that the Hecke algebras $\mathbf{T}_{Q \cup Q^{\prime}}^{\alpha-\text { new }}$ are Gorenstein for any subset $\alpha$ of $Q$. (In [H], Gorensteinness results are proved for the Hecke algebras that arise from action on certain Tate modules: these imply (using [DT]) Gorensteinness of the Hecke algebras considered here. For the result of $[\mathrm{H}]$ the fact that $\bar{\rho}\left(\right.$ Frob $\left._{q_{i}}\right)$ is not a scalar for $q_{i} \in Q$ is crucial, and the technical assumption that $\bar{\rho}$ is not finite at (at least) 2 primes is needed.) Using

- level-raising methods of [DT] and [DT1] (that prove the analog of the Ihara-Ribet lemma in the setting of Shimura curves) and again invoking the isomorphism $\mathbf{T}_{Q}^{\prime \alpha \cup \beta-\text { new }} \simeq \mathbf{T}_{Q}^{\alpha-\text { new }}$

- a simple Galois cohomology computation (see Section 4.2 of [DR])

- and the numerical isomorphism criterion of Wiles (Theorem 3)

it is quite standard (after Section 2 of $[\mathrm{W}]$ ) to deduce the following result from the isomorphism $\pi^{\prime}: R_{Q}^{Q-\text { new }} \simeq \mathbf{T}_{Q}^{Q-\text { new }}$. We skip its proof in this sketch.

Corollary 3 We have an isomorphism $R_{Q \cup Q^{\prime}}^{Q-\text { new }} \simeq \mathbf{T}_{Q \cup Q^{\prime}}^{Q-\text { new }}$.

For any subset $\alpha$ of $Q$, we denote by $\pi$ the morphism $\mathbf{T}_{Q \cup Q^{\prime}}^{\alpha-\text { new }} \rightarrow W(k)$ that sends $T_{r}$ to $\pi^{\prime}\left(T_{r}\right)$ for $r \notin Q^{\prime}$, but sends $T_{r}$ to the unique eigenvalue of $\rho_{Q}^{Q-\text { new }}\left(\right.$ Frob $\left._{r}\right)$ that is congruent to either plus or minus 1 for $r \in Q^{\prime}$. For any subset $\alpha$ of $Q$ that does not contain $q_{i}$, we denote by $\pi_{i}$ the morphism 
$\mathbf{T}_{Q \cup Q^{\prime}}^{\alpha-\text { new }} \rightarrow W(k)$, that arises from the isomorphism $\pi_{i}^{\prime}: \mathbf{T}_{Q_{i}}^{Q_{i}-\text { new }} \simeq W(k)$ of Proposition 4 , that sends $T_{r}$ to $\pi_{i}^{\prime}\left(T_{r}\right)$ for $r \notin Q^{\prime} \cup\left\{q_{i}\right\} \backslash\left\{q_{i}^{\prime}\right\}$ and sends $T_{r}$ to the unique eigenvalue of $\rho_{Q_{i}}^{Q_{i} \text {-new }}\left(\right.$ Frob $\left._{r}\right)$ that is congruent to either plus or minus 1 for $r \in Q^{\prime} \cup\left\{q_{i}\right\} \backslash\left\{q_{i}^{\prime}\right\}$.

Starting from Corollary 3, and using the morphisms $\pi_{i}$ for $i=1, \cdots, n$, Theorem 3, Proposition 2, we briefly indicate how we deduce the isomorphism $R_{Q \cup Q^{\prime}} \simeq \mathbf{T}_{Q \cup Q^{\prime}}$. To do this inductively it suffices to prove that $R_{Q \cup Q^{\prime}}^{Q_{s} \text { new }} \simeq$ $\mathbf{T}_{Q \cup Q^{\prime}}^{Q_{s} \text { new }}$ implies $R_{Q \cup Q^{\prime}}^{Q_{s+1}-\text { new }} \simeq \mathbf{T}_{Q \cup Q^{\prime}}^{Q_{s+1} \text { new }}$ where $Q_{s}=\left\{q_{1}, \cdots, q_{n-s}\right\}$ and $s$ ranges over 0 to $n-1$. As in the previous section to do this it will suffice to prove the following claim:

Claim: The ideal $\left(\pi\left(\operatorname{Ann}_{\mathbf{T}_{Q \cup Q^{\prime}}^{Q_{s+1}-\text { new }}}(\operatorname{ker}(\pi))\right)\right)$ of $W(k)$ is contained in

$$
\left(p^{m_{n-s}}\right)\left(\pi\left(\operatorname{Ann}_{\mathbf{T}_{Q \cup Q^{\prime}}^{Q_{s-n}}}(\operatorname{ker}(\pi))\right)\right)
$$

To prove the claim the two ingredients are the morphism $\pi_{n-s}: \mathbf{T}_{Q \cup Q^{\prime}}^{Q_{s+1} \text { new }} \rightarrow$ $W(k)$ which does not factor through $\mathbf{T}_{Q \cup Q^{\prime}}^{Q_{s}-\text { new }}$ by construction, and the result of $[\mathrm{H}]$ that $\mathbf{T}_{Q \cup Q^{\prime}}^{Q_{s}-\text { new }}$ and $\mathbf{T}_{Q \cup Q^{\prime}}^{Q_{s+1}-\text { new }}$ are Gorenstein. For ease of notation set $\mathbf{T}^{\prime}=\mathbf{T}_{Q \cup Q^{\prime}}^{Q_{s+1}-\text { new }}$ and $\mathbf{T}=\mathbf{T}_{Q \cup Q^{\prime}}^{Q_{s}-\text { new }}$ and denote the natural map $\mathbf{T}^{\prime} \rightarrow \mathbf{T}$ by $\beta$.

We justify the claim using these 2 ingredients: we owe the following argument to the referee. Because $\mathbf{T}$ and $\mathbf{T}^{\prime}$ Gorenstein, we have that

$$
\pi \beta\left(\operatorname{Ann}_{\mathbf{T}^{\prime}}(\operatorname{ker}(\pi \beta))\right)=\pi \beta\left(\operatorname{Ann}_{\mathbf{T}^{\prime}}(\operatorname{ker}(\beta))\right) \pi\left(\operatorname{Ann}_{\mathbf{T}}(\operatorname{ker}(\pi))\right)
$$

as ideals of $W(k)$. Choose a $x \in \operatorname{ker}(\beta)$ such that $\pi_{n-s}(x) \neq 0$ (this exists as $\pi_{n-s}$ does not factor through $\left.\mathbf{T}\right)$. Consider $y \in \operatorname{Ann}_{\mathbf{T}^{\prime}}(\operatorname{ker}(\beta))$. Then as $x y=0$ this implies $\pi_{n-s}(x y)=0$, which implies $\pi_{n-s}(y)=0$, which implies $\pi \beta(y) \in p^{m_{n-s}} W(k)$ as $\pi_{n-s}$ and $\pi$ are congruent mod $p^{m_{n-s}}$ which proves the claim.

\section{Remarks:}

- It follows from [B] that the isomorphism of complete intersections $R_{Q} \simeq$ $\mathbf{T}_{Q}$ implies the isomorphism of complete intersections $R_{Q}^{\alpha-\text { new }} \simeq \mathbf{T}_{Q}^{\alpha \text {-new }}$ for each subset $\alpha$ of $Q$ and hence in particular that $\mathbf{T}_{Q}^{\alpha-\text { new }}$ is Gorenstein. Thus the Gorenstein property of new quotients is a consequences 
of $R_{Q} \simeq \mathbf{T}_{Q}$-type theorems which in turn can be proved (see the previous section) without knowing a priori such a property. In the method of this section on the other hand we needed to use the Gorenstein property of new quotients to prove $R_{Q} \simeq \mathbf{T}_{Q}$-type theorems.

- The referee had pointed out that the arguments of this section in an earlier version had a gap as they invoked a priori Gorenstein properties of new-quotients of Hecke algebras that had not been justified. The result of $[\mathrm{H}]$ fills this gap.

\section{Acknowledgements}

The author would like to express his gratitude to Ravi Ramakrishna for making available $[R]$ and $[R 1]$ while still in preprint form and for pointing out the striking consequence (Theorem 1$]$ of this paper) of the method of [R1] that was the main inspiration for this work. He would also like to thank Fred Diamond and Richard Taylor for helpful conversations, and Ken Ribet for helpful correspondence. He would like to thank Gebhard Böckle and David Helm for writing appendices to the paper. This work germinated during a visit to Brandeis University in the Fall of 1999 and he would like to thank Fred Diamond for the invitation. He would like to thank the referee for a careful reading and helpful critique of the article. Finally he would like to acknowledge the debt this article owes to the beautiful ideas of Ken Ribet and Andrew Wiles.

\section{References}

[B] Böckle, G., On the isomorphism $R_{\emptyset} \rightarrow T_{\emptyset}$, appendix to this paper.

[Ca] Carayol, H., Formes modulaires et représentations galoisiennes avec valeurs dans un anneau local complet, in p-adic monodromy and the Birch and Swinnerton-Dyer conjecture (Boston, MA, 1991), 213-237, Contemp. Math., 165, AMS, 1994.

[Ca1] Carayol, H., Sur les représentations $\ell$-adiques associées aux formes modulaires de Hilbert, Annales de l'Ecole Norm. Sup. 19 (1986), 409-468.

[deS] de Shalit, E., Deformation rings and Hecke rings, in [FLT]. 
[D] Diamond, F., The Taylor-Wiles construction and multiplicity one, Invent. Math. 128 (1997), no. 2, 379-391.

[DDT] Darmon, H., Diamond, F., Taylor, R., Fermat's last theorem, Current developments in mathematics, 1995 (Cambridge, MA), 1-154, Internat. Press, Cambridge, MA, 1994.

[DR] Diamond, F., Ribet, K., $\ell$-adic modular deformations and Wiles's "Main Conjecture", in [FLT].

[DT] Diamond, F., Taylor, R., Lifting modular mod l representations, Duke Math. J. 74 (1994), no. 2, 253-269.

[DT1] Diamond, F., Taylor, R., Nonoptimal levels for mod $\ell$ modular representations of $\operatorname{Gal}(\overline{\mathbf{Q}} / \mathbf{Q})$, Invent. Math. 115 (1994), 435-462.

[FLT] Modular forms and Fermat's last theorem, edited by Gary Cornell, Joseph H. Silverman and Glenn Stevens. Springer-Verlag, New York, 1997.

[G] Grothendieck, A., Exposé IX, SGA7, SLNM 288, 1972.

[GS] Greenberg, R., Stevens, G., p-adic L-functions and p-adic periods of modular forms, Invent. Math. 111 (1993), no. 2, 407-447.

[H] Helm, D., The Gorenstein property for new quotients, appendix to this paper.

[KR] Khare, C., Ramakrishna, R., Finiteness of Selmer groups and deformation rings, preprint.

[Ma] Mazur, B., An introduction to the deformation theory of Galois representations, in [FLT].

[R] Ramakrishna, R., Lifting Galois representations, Invent. Math. 138 (1999), 537-562.

[R1] Ramakrishna, R., Deforming Galois representations and the conjectures of Serre and Fontaine-Mazur, to appear in Annals of Math.

[RT] Ribet, K., Takahashi, S., Parametrizations of elliptic curves by Shimura curves and by classical modular curves, in Elliptic curves and modular forms (Washington, DC, 1996). Proc. Nat. Acad. Sci. U.S.A. 94 (1997), no. 21, 11110-11114. 
[Ri] Ribet, K., On modular representations of $\mathrm{Gal}(\overline{\mathbf{Q}} / \mathbf{Q})$ arising from modular forms, Invent. Math. 100 (1990), no. 2, 431-476.

[Ri1] Ribet, K., Report on mod $\ell$ representations of $\mathrm{Gal}(\overline{\mathbf{Q}} / \mathbf{Q})$, in Motives, Proc. Sympos. Pure Math. 55, Part 2 (1994), 639-676.

[Ri2] Ribet, K., Congruence relations between modular forms, Proc. International Cong. of Math. (1983), pp 503-514.

[Ri3] Ribet, K., Galois action on division points of Abelian varieties with real multiplications, Amer. J. Math. 98 (1976), no. 3, 751-804.

[Ri4] Ribet, K., Multiplicities of Galois representations in Jacobians of Shimura curves, Israel Math. Conf. Proc., 3, Weizmann, Jerusalem, 1990.

[S] Serre, J-P., Sur les représentations modulaires de degré 2 de Gal $(\overline{\mathbf{Q}} / \mathbf{Q})$, Duke Math. J. 54 (1987), 179-230.

[TW] Taylor, R., Wiles, A., Ring-theoretic properties of certain Hecke algebras, Ann. of Math. (2) 141 (1995), no. 3, 553-572.

[W] Wiles, A., Modular elliptic curves and Fermat's last theorem, Ann. of Math. (2) 141 (1995), no. 3, 443-551.

Address of author: School of Mathematics, TIFR, Homi Bhabha Road, Mumbai 400 005, INDIA: shekhar@math.tifr.res.in

Dept. of Math, University of Utah, 155 S 1400 E, Salt Lake City, UT 84112, USA: shekhar@math.utah.edu 